\title{
Comment on "A periodic grain consolidation model of porous media" [Phys. Fluids A 1, 38 (1989)]
}

Cite as: Phys. Fluids 31, 109101 (2019); https://doi.org/10.1063/1.5116700

Submitted: 27 June 2019 . Accepted: 30 July 2019. Published Online: 11 October 2019

Siarhei Khirevich (D), and Tadeusz W. Patzek (D)

AlP Author Services

English Language Editing

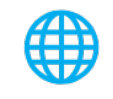




\title{
Comment on "A periodic grain consolidation model of porous media" [Phys. Fluids A 1, 38 (1989)]
}

\author{
Cite as: Phys. Fluids 31, 109101 (2019); doi: 10.1063/1.5116700 \\ Submitted: 27 June 2019 - Accepted: 30 July 2019 • \\ Published Online: 11 October 2019
}

Siarhei Khirevich $^{\text {) }}$ (D) and Tadeusz W. Patzek

\section{AFFILIATIONS}

Ali I. Al-Naimi Petroleum Engineering Research Center, King Abdullah University of Science and Technology, Thuwal, Saudi Arabia

a) Electronic mail: siarhei.khirevich@kaust.edu.sa

\begin{abstract}
In this document, we correct the friction coefficient values presented in Table III in a study by Larson and Higdon ["A periodic grain consolidation model of porous media," Phys. Fluids A 1, 38 (1989)]. The authors addressed the problem of Stokes flow through periodic arrays of (non)overlapping spheres and determined the friction coefficients. It appears that the volume of the overlapping region of spheres was not taken into account, which affected the total solid concentration and systematically biased the corresponding friction coefficient values. We correct the sphere concentration and friction coefficients, and validate our approach with lattice-Boltzmann simulations. The suggested correction is valid in the case of overlapping spheres only, when the volume of the overlapping region is positive.
\end{abstract}

Published under license by AIP Publishing. https://doi.org/10.1063/1.5116700

\section{GOVERNING EQUATIONS}

Following the work of Hill et al. ${ }^{1}$ or van der Hoef et al., ${ }^{2}$ under stationary flow conditions, the pressure gradient $\nabla P$ exerted on an array of $n_{\text {sp }}$ fixed solid spheres in the domain of volume $V_{\text {domain }}$ can be described as follows:

$$
|\nabla P|=|f| \frac{n_{\mathrm{sp}}}{V_{\text {domain }}},
$$

where $f$ is the average drag force acting on the spheres. The volumetric sphere concentration, $c$ (or the solid volume fraction), can be expressed as

$$
c\left(d_{\mathrm{sp}}\right)=\frac{n_{\mathrm{sp}}}{V_{\text {domain }}} \frac{\pi}{6} d_{\mathrm{sp}}^{3}
$$

where $d_{\text {sp }}$ is the sphere diameter.

Let us introduce the friction coefficient $K$,

$$
K=\frac{|f|}{3 \pi \mu d_{\mathrm{sp}}|v|}
$$

where the denominator is the Stokes drag force acting on a single sphere in an unbounded fluid and $|v|$ is the average flow velocity. Combining (1)-(3), we obtain

$$
K=\frac{|\nabla P| V_{\text {domain }}}{n_{\mathrm{sp}}} \frac{1}{3 \pi \mu d_{\mathrm{sp}}|v|}=\frac{d_{\mathrm{sp}}^{2}|\nabla P|}{18 \pi \mu|v| c} .
$$

\section{OVERLAPPING SPHERES: CORRECTION TO CONCENTRATION}

Equation (2) can be formally applied to spheres of any size, but when spheres overlap, their calculated volume concentration is incorrect. Equation (2) was used by Larson and Higdon ${ }^{3}$ to calculate sphere concentrations in their Table III. Despite this statement by the authors, "....For overlapping spheres, the volume fraction is given by a slightly more complicated expression that accounts for the volume of the spherical caps in the overlapping region ...," it seems that the volume of the overlapping region was not taken into account. Therefore, their values of $K$ for overlapping spheres were also affected. When spheres overlap, the correct value of concentration $c$ in Eq. (4) should be calculated as

$$
c^{*}=\frac{n_{\mathrm{sp}}}{V_{\text {domain }}}\left(\frac{\pi}{6} d_{\mathrm{sp}}^{3}-n_{\text {cap }} V_{\text {cap }}\right),
$$

where $n_{\text {cap }}$ is the number of spherical caps (or sphere contacts) per each sphere in a unit cell and $V_{\text {cap }}$ is the volume of each spherical 
cap. A spherical cap is formed when two touching spheres begin to overlap one another after a further increase in their diameter(s). Assuming two centers of equal-diameter spheres are separated by distance $s$, i.e., $d_{\mathrm{sp}}=s$ when the spheres touch, the spherical cap volume is

$$
V_{\text {cap }}=\frac{\pi}{3}\left(\frac{d_{\mathrm{sp}}^{3}}{4}-\frac{3}{8} s d_{\mathrm{sp}}^{2}+\frac{s^{3}}{8}\right), \text { for } d_{\mathrm{sp}} \geq s
$$

For the three considered packing types (fcc, bcc, and sc), $n_{\mathrm{sp}}^{\mathrm{fcc}}$ $=4, n_{\mathrm{sp}}^{\mathrm{bcc}}=2$, and $n_{\mathrm{sp}}^{\mathrm{sc}}=1 ; V_{\mathrm{domain}}^{\mathrm{fcc}}=(s \sqrt{2})^{3}, V_{\text {domain }}^{\mathrm{bcc}}=(s 2 / \sqrt{3})^{3}$, and $V_{\text {domain }}^{\text {sc }}=s^{3}$; and $n_{\text {cap }}^{\mathrm{fcc}}=12, n_{\text {cap }}^{\mathrm{bcc}}=8$, and $n_{\text {cap }}^{\text {sc }}=6$.

Using the value of concentration $c_{\text {original }}$ from Table III in the original study, ${ }^{3}$ one can obtain the corresponding diameter $d_{\mathrm{sp}}^{\text {original }}$ after finding the smallest positive root of the following cubic equation:

$$
\begin{gathered}
\left(\frac{1}{2 n_{\text {cap }}}-\frac{1}{4}\right)\left(d_{\text {sp }}^{\text {original }}\right)^{3}+\frac{3}{8} s\left(d_{\text {sp }}^{\text {original }}\right)^{2} \\
-\left(\frac{s^{3}}{8}+\frac{3 V_{\text {domain }} c_{\text {original }}}{\pi n_{\text {sp }} n_{\text {cap }}}\right)=0
\end{gathered}
$$

The value of $d_{\mathbf{s p}}^{\text {original }}$ can be used to calculate $c\left(d_{\mathbf{s p}}^{\text {original }}\right)$ from Eq. (2). Then, the following correction should be applied to the values of $K^{\text {original }}$ for overlapping spheres:

$$
K^{\text {corrected }}=K^{\text {original }} \frac{c\left(d_{\mathrm{sp}}^{\text {original }}\right)}{c_{\text {original }}} .
$$

\section{ALTERNATIVE DETERMINATION OF THE FRICTION COEFFICIENT AND VALIDATION}

To validate Eq. (8), we determined the friction coefficients using an alternative approach presented in the study of Khirevich and Patzek. ${ }^{5}$ Their approach is based on the lattice-Boltzmann method (LBM) simulations and extrapolation toward an infinitely high resolution of the discretization. For the extrapolation, the friction coefficient values were obtained using the discretization resolutions of 290 lattice nodes per sphere diameter or higher and LBM parameter $\Lambda=0.05$. Such a simulation setup provides values of $K$ with accuracy of $10^{-3}$ or better. $^{5}$ Results of LBM simulations and extrapolation are demonstrated in Fig. 1.

Table I presents both the correct (column A) and incorrect (column B) values of $K$ based on the LBM approach. The original $K$ values of Larson and Higdon ${ }^{3}$ in column $C$ are similar to those in column B. The friction coefficient values corrected with Eq. (8) are similar to the LBM results in column A.

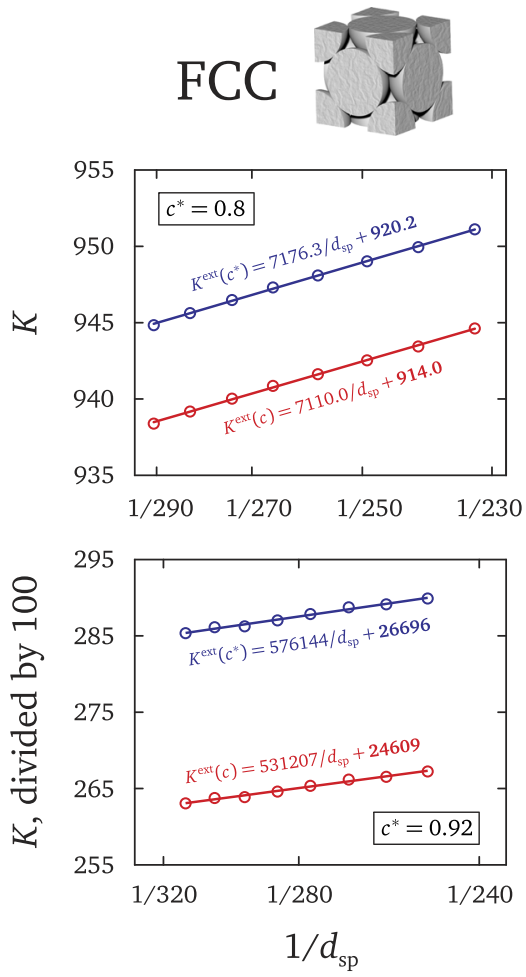

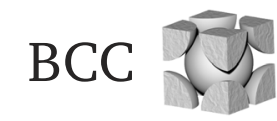
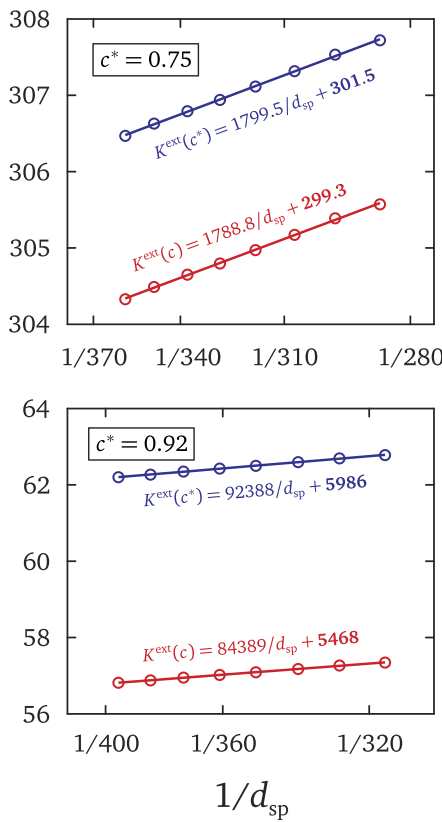
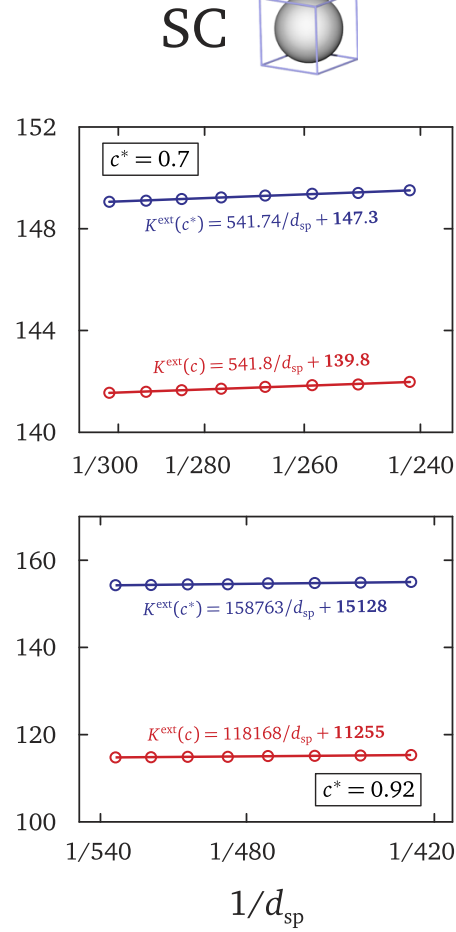

FIG. 1. Results of the lattice-Boltzmann simulations using the extrapolation approach presented previously. ${ }^{5}$ Using average flow velocity, friction coefficient $K$ for overlapping spheres can be calculated according to Eq. (4) using the correct concentration value from Eq. (5) (blue color) or the incorrect one from Eq. (2) (red color). Both approaches can be used to find accurate values of $K$ using linear extrapolation. 
TABLE I. Friction coefficients determined for various packing types and concentrations. Columns A and B: values of $K$ determined via the extrapolation approach from the study of Khirevich and Patzek ${ }^{5}$ using $c^{*}$ and $c$, respectively. C: Original values from Larson and Higdon. ${ }^{3} \mathrm{D}$ : Corrected values from column $\mathrm{C}$ using Eq. (8). The italic value indicates a typo in the exponent in the original study of Larson and Higdon, ${ }^{3}$ where $K_{\mathrm{bcc}, \mathrm{c}=0.92}^{\text {original }}=5.47 \times 10^{4}$.

\begin{tabular}{lccccc}
\hline \hline $\begin{array}{l}\text { Packing } \\
\text { type }\end{array}$ & $\begin{array}{c}\text { Packing } \\
\text { concentration }\end{array}$ & $\begin{array}{c}\text { A: Khirevich, } \\
K \text { using } c^{*}\end{array}$ & $\begin{array}{c}\text { B: Khirevich, } \\
K \text { using } c\end{array}$ & $\begin{array}{c}\text { C: Larson, } \\
K^{\text {original }}\end{array}$ & $\begin{array}{c}\text { D: Larson, } \\
K^{\text {corrected }}, \text { Eq. (8) }\end{array}$ \\
\hline fcc & 0.8 & 920.2 & 914.0 & 913.8 & 920.2 \\
bcc & 0.75 & 301.5 & 299.3 & 299.2 & 301.3 \\
sc & 0.7 & 147.3 & 139.8 & 139.8 & 147.2 \\
\hline $\mathrm{fcc}$ & 0.92 & $2.67 \times 10^{4}$ & $2.46 \times 10^{4}$ & $2.48 \times 10^{4}$ & $2.69 \times 10^{4}$ \\
$\mathrm{bcc}$ & 0.92 & $5.99 \times 10^{3}$ & $5.47 \times 10^{3}$ & $5.47 \times 10^{3}$ & $5.99 \times 10^{3}$ \\
$\mathrm{sc}$ & 0.92 & $1.51 \times 10^{4}$ & $1.13 \times 10^{4}$ & $1.20 \times 10^{4}$ & $1.61 \times 10^{4}$ \\
\hline \hline
\end{tabular}

For the sake of completeness, we comment on the limits of applicability of Eq. (5). With increasing $d_{\text {sp }}$, new sphere-to-sphere contacts are introduced and Eq. (5) needs further modification. For fcc packing, this will occur at $d_{\mathrm{sp}}^{\mathrm{bcc}}>1.15 s$ and $c>0.96$. For bcc packing, new contacts will appear at $d_{\mathrm{sp}}^{\mathrm{bcc}}>s 2 / \sqrt{3}$ and $c>0.939$. For sc packing, no new contacts will appear until the geometry becomes impermeable at $d_{\mathrm{sp}}^{\mathrm{sc}}>\sqrt{2}$ and $c>0.965$.

\section{ACKNOWLEDGMENTS}

We are grateful for the allocation of computational resources by the Supercomputing Laboratory at King Abdullah University of Science and Technology (KAUST) in Thuwal, Saudi Arabia.

\section{REFERENCES}

${ }^{1}$ R. J. Hill, D. L. Koch, and A. J. C. Ladd, "The first effects of fluid inertia on flows in ordered and random arrays of spheres," J. Fluid Mech. 448, 213-241 (2001).

${ }^{2}$ M. A. van der Hoef, R. Beetstra, and J. A. M. Kuipers, "Lattice-Boltzmann simulations of low-Reynolds-number flow past mono- and bidisperse arrays of spheres: Results for the permeability and drag force," J. Fluid Mech. 528, 233-254 (2005).

${ }^{3}$ R. E. Larson and J. J. L. Higdon, "A periodic grain consolidation model of porous media," Phys. Fluids 1, 38-46 (1989).

${ }^{4} \mathrm{~J}$. W. Harris and H. Stöcker, Handbook of Mathematics and Computational Science (Springer, 1998).

${ }^{5}$ S. Khirevich and T. Patzek, "Behavior of numerical error in pore-scale lattice Boltzmann simulations with simple bounce-back rule: Analysis and highly accurate extrapolation," Phys. Fluids 30, 093604 (2018). 